\title{
Benzyl Farnesyl Amine Mimetics are Potent Inhibitors of the Sterol Biosynthesis Pathway in Leishmania Amazonensis Leading to Oxidative Stress, Growth Arrest, and Ultrastructural Alterations
}

\section{Sara Teixeira Macedo-Silva}

Universidade Federal do Rio de Janeiro

\section{Gonzalo Visbal}

Instituto Nacional de Metrologia, Qualidade e Tecnologia

Gabrielle Frizzo Souza

UNICAMP

Mayara Roncaglia dos Santos

UNICAMP

Simon B. Cämmerer

UNICAMP

\section{Wanderley de Souza}

Universidade Federal do Rio de Janeiro

Juliany Cola Fernandes Rodrigues ( $\nabla$ juliany.rodrigues@caxias.ufri.br )

Universidade Federal do Rio de Janeiro

\section{Research Article}

Keywords: Sterol biosynthesis pathway, squalene synthase inhibitors, Leishmania, oxidative stress, chemotherapy, cell death

Posted Date: January 3rd, 2022

DOI: https://doi.org/10.21203/rs.3.rs-1181920/v1

License: (9) This work is licensed under a Creative Commons Attribution 4.0 International License. Read Full License 


\section{Abstract}

Leishmaniasis is a neglected disease caused by protozoan parasites of the Leishmania genus spread around the world. Benzyl farnesyl amine mimetics are known class of compounds selectively designed to inhibit the squalene synthase (SQS) enzyme that catalyzes the first committed reaction on the sterol biosynthesis pathway. Herein, we studied seven new benzyl farnesyl amine mimetics (SBC 37 - 43) against Leishmania amazonensis. After the first initial screening of cell viability, two inhibitors (SBC 39 and SBC 40) were selected for further studies. Against intracellular amastigotes, SBC 39 and SBC 40 presented selectivity indexes of 117.7 and 180, respectively, indicating that they are highly selective. Analyses of free sterol showed that SBC 39 and SBC 40 inhibit two enzymes, sterol $\Delta^{8} \rightarrow \Delta^{7}$ isomerase and SQS, resulting in depletion of endogenous 24-methyl sterols. Physiological analysis and electron microscopy revealed three main alterations: 1 ) in the mitochondrion ultrastructure and function; 2) the presence of lipid bodies and autophagosomes; and 3) the appearance of projections in the plasma membrane and extracellular vesicles inside the flagellar pocket. In conclusion, our results support the notion that benzyl farnesyl amine mimics have a potent effect against Leishmania amazonensis and should be an interesting novel pharmaceutical lead for the development of new chemotherapeutic alternatives to treat leishmaniasis.

\section{Introduction}

Leishmaniasis is an endemic neglected disease caused by several species of Leishmania genus. The leishmaniasis transmission was reported in a total of 98 countries and 3 territories on 5 continents. The estimated world prevalence for all clinical manifestations of the disease is 12 million, with 58,000 of visceral leishmaniasis (VL) cases and 220,000 cutaneous leishmaniasis (CL) cases per year [1,2]. In the New World, multiple species, including $L$. amazonensis and $L$. braziliensis, are the causative agents of the CL. Furthermore, $L$. amazonensis can also cause mucocutaneous leishmaniasis (MCL), which results in a progressive destruction of the naso-oropharyngeal mucosa, and diffuse cutaneous leishmaniasis (DCL), the most severe cutaneous form of the disease that is characterized by a diffuse infiltration in the skin of the patients and highly resistant to all kind of chemotherapy available [3, 4]. Besides of the severe clinical manifestations of the leishmaniasis, there are few drugs available for its treatment.

Pentavalent antimonials are the first choice of treatment for more than 70 years in some countries around the world, despite the parenteral route of administration, high toxicity and cost [5]. Second-line treatments are based on the use of amphotericin B formulations and pentamidine. In some countries in Asia, Africa, and Europe, Miltefosine (Impavido) is currently the first line-treatment [5, 6]. Among the chemotherapeutic agents available, miltefosine is the only orally available treatment, however it is teratogenic and not indicated for woman of fertile age $[7,8]$. None of the available drugs can be considered ideal due to their high toxicity, long duration of treatment, and severe side effects, which often lead to treatment abandonment. Furthermore, these treatments do not be eliminate the parasite completely in the infected individuals $[9,10]$. 
Trypanosomatids and fungi have an endogenous requirement of ergosterol and other 24-alkylated sterols for growth and survival, which are absent in mammal cells. Thus, the enzymes of sterol biosynthesis pathway are interesting targets for new treatments and several works have shown the effect of different sterol biosynthesis inhibitors (SBIs) in trypanosomatids [11-17]. Benzyl farnesyl amine mimetics are one class of selective inhibitors of the squalene synthase (SQS), an important enzyme that catalyzes the condensation of two molecules of farnesyl pyrophosphate to produce squalene. This is the first committed step in the sterol biosynthesis, and its inhibition does not affect the synthesis of isoprenoids that are also important molecules for eukaryotic cells. For years, the research for specific squalene synthase inhibitors (SQSi) has involved intensive efforts of industrial and academic investigators, because of the potential use of these inhibitors in the treatment of coronary heart disease and hypercholesterolemia [18-20].

Among the selective inhibitors of the SQS [21-23], we early investigated the antiprotozoal potential of $\mathrm{BPQ}-\mathrm{OH}$ (3-biphenyl-4-yl-3-hydroxyquinuclidine), an arylquinuclidine, with significant biological activity against $T$. cruzi and L. mexicana, resulting in growth inhibition, cell lysis and complete depletion of endogenous squalene and 24-methyl sterols [27]. Against L. amazonensis, BPQ-OH presented a potent growth inhibition effect against both developmental stages of the parasite leading to several changes in the ultrastructure of promastigotes [28]. Furthermore, other two quinuclidine derivatives, E-5700 and ER119884, also showed potent antiproliferative effects against T. cruzi [30] and L. amazonensis, also in combination with C14a-demethylase inhibitors [azoles] [17, 23].

In this work, the effect of another class of selective SQS inhibitors, novel mimetics of benzyl farnesyl amine, were evaluated against Leishmania amazonensis. Several aspects of the anti-Leishmania activity of these compounds were investigated in different times of treatment, such as antiproliferative, ultrastructural and biochemical effects. Moreover, we found one derivative that is among the most selective compounds for the parasite with low toxicity for the mammalian cells.

\section{Material And Methods \\ 2.1. Ethics statements.}

The experiments using BALB/c mice to isolate macrophages and maintained Leishmania parasite were approved by the Ethics Committee for Animal Experimentation of the Health Sciences Centre, Federal University of Rio de Janeiro (Protocols n. IBCCF 096/097/106), according to the Brazilian federal law (11.794/2008, Decreto no 6.899/2009). All animals received humane care in compliance with the "Principles of Laboratory Animal Care" formulated by the National Society for Medical Research, and the "Guide for the Care and Use of Laboratory Animals" prepared by the National Academy of Sciences, USA.

\subsection{Parasites}


The MHOM/BR/75/Josefa strain of L. amazonensis used in this study was gently provided by the Leishmania Collection of the Instituto Oswaldo Cruz (code IOCL 0071-FIOCRUZ). It has been maintained via inoculation into the base of BALB/c mouse tails. Amastigotes were obtained from the lesions of infected mice and transformed into promastigotes that were maintained in Warren's medium [brain heart infusion plus hemin and folic acid] [24] supplemented with $10 \%$ fetal bovine serum (FBS) at $25^{\circ} \mathrm{C}$. Infective metacyclic promastigotes were used to obtain intracellular amastigotes in macrophage culture. Firstly, peritoneal macrophages from BALB/c mice were harvested by washing them with Hanks' solution and plated in 24-well tissue culture chamber slides, allowing them to adhere to the slides for $24 \mathrm{~h}$ in RPMI medium (Gibco) supplemented with $10 \% \mathrm{FBS}$ at $37^{\circ} \mathrm{C}$ in $5 \% \mathrm{CO}_{2}$. After this, adherent macrophages were infected with metacyclic promastigotes at a macrophage-to-parasite ratio of $1: 10$ at $35^{\circ} \mathrm{C}$ in $5 \% \mathrm{CO}_{2}$ for 2 $\mathrm{h}$. These cultures were maintained for $24 \mathrm{~h}$ in RPMI medium supplemented with $10 \%$ FBS for the assays with intracellular amastigotes.

\subsection{Drugs.}

The benzyl farnesyl amine mimetics, SBCs 37 - 43, were prepared by chemical synthesis at IQ-UNICAMP according to an experimental procedure previously described by Cämmerer and Souza [25]. Structures of those $N$-[4-[benzyloxy] benzyl]-benzene-methaneamine derivatives, SBCs $37-43$, are displayed in scheme 1. These compounds have been recently reported to exhibit significant biological activity against intracellular amastigotes of Trypanosoma cruzi [25]. Compounds were used as hydrochloride salts, which were purified by one recrystallization from analytical grade ethanol and dried in high vacuum at room temperature.

\subsection{Cell viability and cytotoxicity assays}

For primary screening of the antileishmanial effects of the SBCs 37-43, we evaluated the cell viability and cytotoxicity effects in L. amazonensis promastigotes and peritoneal macrophages by CellTiter $96 \AA$ Aqueous MTS Assay (Promega, United States) $[16,29]$. For analysis in promastigotes, we started the culture at cell density of $1 \times 10^{6} \mathrm{cells} / \mathrm{ml}$ in Warren's medium supplemented with $10 \%$ FBS. After $24 \mathrm{~h}$, different concentrations of SBC37-43 were added to the cultures. Cell viability and the cytotoxic effects were measured at 24,48 , and $72 \mathrm{~h}$ of treatment, when all groups, including untreated, were transferred to clear 96 -well plate in triplicate. MTS/PMS assay reaction was quantified by optical density measurement at $490 \mathrm{~nm}$ in a microplate reader and SpectraMax $\mathrm{M}_{2} / \mathrm{M}_{2}{ }^{\mathrm{e}}$ spectrofluorometer (Molecular Devices, United States). As a negative control, parasites were fixed with $0.4 \%$ nascent formaldehyde for $10 \mathrm{~min}$ at room temperature before the incubation. Cytotoxicity effects of SBCs 37 - 43 in murine macrophages were also evaluated using the same MTS/PMS assay reaction described above. Murine macrophages were obtained from BALB/c mouse after washing with Hanks's solution and cultivated in a clear 96-well plate with RPMI medium supplemented with $10 \% \mathrm{FBS}$ and maintained at $37^{\circ} \mathrm{C}$ in $5 \% \mathrm{CO}_{2}$. After $24 \mathrm{~h}$ of cultivation, SBCs 37 - 43 were added at different concentrations. Macrophages viability was measured at 24,48 , and $72 \mathrm{~h}$ of treatment. MTS/PMS assay reaction was also quantified by optical density measurement at $490 \mathrm{~nm}$ in a microplate reader and SpectraMax $\mathrm{M}_{2} / \mathrm{M}_{2}{ }^{\mathrm{e}}$ spectrofluorometer (Molecular 
Devices, United States). The cytotoxicity concentration to reduce $50 \%$ of viable macrophages $\left(\mathrm{CC}_{50}\right)$ was determined.

\subsection{Growth inhibition of promastigotes and amastigotes of L. amazonensis}

After the evaluation of the cell viability and cytotoxic effects by MTS assay in promastigote forms, we also analyzed the effects of the SBCs 37 - 43 in the growth of promastigotes. For this, promastigote cultures were initiated at a cell density of $1.0 \times 10^{6}$ cells $/ \mathrm{ml}$. After $24 \mathrm{~h}$ of growth, SBCs $37-43$ were added at different concentrations from concentrated stock solutions, and cell densities were evaluated daily over $96 \mathrm{~h}$ of growth using a Neubauer chamber. Based on the analysis of $\mathrm{CC}_{50}$ and $\mathrm{IC}_{50}$ in promastigotes, and the cytotoxic effects in murine macrophages, three of the benzyl farnesyl amine mimetics (SBCs 37, 39 and 40) were chose for evaluation against amastigotes infected macrophages. To evaluate the effects of compounds on L. amazonensis intracellular amastigotes, macrophages were infected as described previously and incubated with different concentrations of compounds after $24 \mathrm{~h}$ of infection. Fresh medium was added daily until 3 days $(24,48$, and 72 h of treatment). After this time, cultures were fixed in Bouin's solution [17], and washed with 70\% ethanol, distilled water and then stained with Giemsa solution for $1 \mathrm{~h}$. The number of intracellular amastigotes was obtained after count in light microscopy. Association indexes ((mean number of parasites internalized X percentage of infected macrophages) / total number of macrophages) were determined and used as a parameter to calculate the percentage of infection for each condition used in this study. The concentration that inhibited $50 \%$ of growth $\left(\mathrm{IC}_{50}\right)$ and selective index (SI) were calculated.

\subsection{Estimation of the mitochondrial transmembrane electric potential}

Mitochondrial transmembrane electric potential $\left(\Delta \psi_{m}\right)$ of the untreated and treated promastigotes was analyzed using the JC-1 fluorochrome (Molecular Probes, United States), a lipophilic and cationic mitochondrial vital dye that accumulates in the mitochondria in response to $\Delta \psi_{m}$, since its fluorescence is considered an indicator of an energized mitochondrial state [15]. JC-1 exits as a J-monomer that in absence of $\Delta \Psi_{m}$ accumulate in low concentration with emission wavelength at $530 \mathrm{~nm}$ (green fluorescence), however, in presence of $\Delta \psi_{m}$ JC-1 accumulated as J-aggregates with emission at $590 \mathrm{~nm}$ (red fluorescence). Parasites were prepared as previously described $[15,16]$. For each sample, $1 \times 10^{7}$ parasites were incubated with $10 \mu \mathrm{g} / \mathrm{mL}$ JC-1 for $25 \mathrm{~min}$, with readings made every minute using a microplate reader and spectrofluorometer SpectraMax M2/M2 (Molecular Devices, United States). Cells were incubated in the presence of $2 \mu \mathrm{M}$ FCCP (a mitochondrial protonophore) during the initial 20 min of experiment as positive control for the mitochondrial membrane depolarization. After 20 min of readings, $2 \mu \mathrm{M}$ FCCP was added at all samples to abolish the $\Delta \psi_{m}$. The relative $\Delta \psi_{m}$ values were obtained calculating the ratio between the reading at $590 \mathrm{~nm}$ and $530 \mathrm{~nm}(590: 530 \mathrm{~nm})$. Experiments were 
independently repeated at least three times in triplicate, and graphic shows the mean and standard deviation of one representative experiment.

\subsection{Evaluation of ROS production}

Intracellular ROS levels were evaluated in control and compound-treated promastigotes as described previously [17]. For this, $3 \times 10^{7}$ promastigotes were harvested, washed twice in $\mathrm{PBS}(\mathrm{pH} 7.2)$ and incubated with $10 \mu \mathrm{g} / \mathrm{ml} \mathrm{H}_{2}$ DCFDA [a cell-permeable green probe; Molecular Probes, United States] in PBS for $1 \mathrm{~h}$ at $25^{\circ} \mathrm{C}$. After $1 \mathrm{~h}$, cells were washed and resuspended in PBS, added in a black 96-well plate, and then analyzed in a microplate reader and spectrofluorometer SpectraMax M2/M2 ${ }^{\mathrm{e}}$ (Molecular Devices, United States), using the pair of $507 \mathrm{~nm}$ and $530 \mathrm{~nm}$ wavelengths as emission and excitation wavelengths, respectively.

\subsection{Lipid bodies accumulation}

For analysis of lipid bodies accumulation, $1.0 \times 10^{7}$ cells were harvested, washed in PBS (pH 7.2), and incubated with $10 \mu \mathrm{g} / \mathrm{ml}$ Nile Red (Sigma, Brazil) for $20 \mathrm{~min}$. After this step, cells were washed twice in PBS, resuspended in $200 \mu \mathrm{l}$ of PBS and then added to a black 96-well plate. Readings were taken in a microplate reader and spectrofluorometer SpectraMax $\mathrm{M} 2 / \mathrm{M}^{\mathrm{e}}$ (Molecular Devices, United States), using the wavelengths 485 and $538 \mathrm{~nm}$ for excitation and emission, respectively.

\subsection{Electron microscopy}

First, control and treated promastigotes and intracellular amastigotes were fixed in $2.5 \%$ glutaraldehyde in $0.1 \mathrm{M}$ cacodylate buffer $(\mathrm{pH}$ 7.2) for $1 \mathrm{~h}$ at room temperature. Second, the samples were postfixed in a solution containing $1 \% \mathrm{OsO}_{4}, 1.25 \%$ potassium ferrocyanide, and $5 \mathrm{mM} \mathrm{CaCl} 2$ in $0.1 \mathrm{M}$ cacodylate buffer (pH 7.2) for $30 \mathrm{~min}$. For scanning electron microscopy, promastigotes were dehydrated in ethanol $(30,50$, 70,90 and $100 \%$ ) and critical point-dried in $\mathrm{CO}_{2}$. After that, samples were sputtered with a thin gold layer, and then observed under a FEI Quanta 250 scanning electron microscope. For transmission electron microscopy, cells were dehydrated in acetone and embedded in epoxy resin. After that, ultrathin sections were stained with uranyl acetate and lead citrate and observed under a Zeiss 900 electron microscope.

\subsection{Electron tomography}

For electron tomography, ribbons of $200 \mathrm{~nm}$ thick serial sections were produced from transmission electron microscopy blocks described above. These ribbons were collected in a formvar-coated copper slot grids. After that, colloidal gold particles $(10 \mathrm{~nm})$ were deposited on both surfaces of the sections, being used as fiducial markers for alignment of the tilted views. Single-axis tilt series $\left( \pm 60^{\circ}\right.$ with $1^{\circ}$ increments) were produced from samples using Xplore3D software and a Tecnai-G2 (FEI Company, Eindhoven, Netherlands) electron microscope operating at $200 \mathrm{kV}$. 3D reconstruction was performed using the IMOD software package [27]. Furthermore, tomogram generation by R-weighted back-projection was performed using ETOMO, and virtual slices were manually segmented using 3DMOD that was also used to produce 3D models. 
2.11. Extraction, separation of neutral lipids and free sterol analysis.

For the analysis of the effects of SBC 39 and SBC 40 on the free sterol composition of the promastigotes, total lipids were extracted from control and drug-treated $L$. amazonensis promastigotes, as described previously $[17,28]$. Neutral lipids were analyzed by MS and mass spectra were obtained by electron ionization (EI) at $70 \mathrm{eV}$ according to the protocol published previously $[17,28]$. The assignment of structures was based on relative chromatographic behaviors, as well as the characteristic fragmentation patterns in MS and by comparison of the mass spectra with those available in the National Institute of Standards and Technology (NIST) Research Library located at the NIST Mass Spectrometry Data Center.

2.12. Calibration for cholesterol and ergosterol determination.

A set of five calibration standards was prepared from the pure standard of cholesterol and ergosterol purchased from Sigma-Aldrich Co. Different calibration solutions were prepared using ethyl acetate as solvent. For the quantification of cholesterol and ergosterol, standards were used at different concentrations of $0.08,0.10,0.25,0.50$ and $1.0 \mathrm{mM}$ to plot the standard curve. From each calibration solution, $1 \mu \mathrm{L}$ was injected (run in triplicate) into the GC-MS system to achieve the regression plot of various concentrations versus their peak area.

\subsection{Statistical analysis}

All the graphics in the figures were created using the means of three independent experiments, and the bars represent the standard deviations of the means. The statistical significance of differences among the groups was assessed using the one-way or two-way analysis of variance (ANOVA) test, followed by Bonferroni's multiple-comparison test in the GraphPad Prisma 5 software. Results were considered statistically significant when $P$ was $<0.05\left({ }^{\star}\right),<0.01\left(^{\star \star}\right)$, and $<0.001\left(^{\star \star \star}\right)$.

\section{Results}

\subsection{The cytotoxic and antiproliferative effects of benzyl farnesyl amine mimetics (SBC 37 - 43) against macrophages and Leishmania amazonensis}

Figure 1 shows the cytotoxic effects of seven benzyl farnesyl amine mimetics (SBC 37 - 43) by MTS/PMS assay in promastigotes of Leishmania amazonensis. Six of them had $\mathrm{CC}_{50}$ lower than $5 \mu \mathrm{M}$ (SBC 37, SBC 39, SBC 40, SBC 41, SBC 42, and SBC 43), indicating a promising effect against $L$. amazonensis. These inhibitors were used to evaluate their potential antiproliferative effects against promastigotes of $L$. amazonensis. Figure 2 shows that the most potent inhibitors were SBC 37, SBC 39, SBC 40, and SBC 43 with $\mathrm{IC}_{50}$ of $557 \mathrm{nM}, 560.05 \mathrm{nM}, 303.78 \mathrm{nM}$, and $536.85 \mathrm{nM}$, respectively, after $72 \mathrm{~h}$ of treatment. 
We also evaluated the cytotoxic effects of the SBCs 37 - 43 against murine macrophages using MTS/PMS assay after $72 \mathrm{~h}$ of treatment. Figure 3 shows that SBC 37, SBC 38, SBC 39, and SBC 40 presented low cytotoxicity to mammalian cells, with $\mathrm{CC}_{50}$ of $33.94 \mu \mathrm{M}, 40.53 \mu \mathrm{M}, 40.65 \mu \mathrm{M}$, and 39.15 $\mu \mathrm{M}$, respectively. Based on the results obtained for macrophage and promastigotes, we decided to evaluate the effects of three of them (SBCs 37, 39 and 40) against intracellular amastigotes. Figure 4 shows the antiproliferative effects of SBC 37, SBC 39, and SBC 40 after $72 \mathrm{~h}$ of treatment, presenting $\mathrm{IC}_{50}$ values of $740.48 \mathrm{nM}, 345.35 \mathrm{nM}$, and $217.5 \mathrm{nM}$, respectively (Fig. 4A-C). Thus, the selectivity index obtained was $45.83,117.7$, and 180 , respectively, after $72 \mathrm{~h}$ of treatment.

\subsection{SBC 39 and SBC 40 alter the morphology of promastigotes}

Scanning electron microscopy revealed important changes in the morphology of promastigotes treated with SBC 39 and SBC 40 for $48 \mathrm{~h}$ (Fig. 5-F). Figure 5A shows a control L. amazonensis promastigote without any alteration in the morphology of cell body, surface and flagellum (Fig. 5A). Treatment with lower concentrations of SBCs induced several alterations such as the presence of parasites rounded and swollen (Fig. 5B, C, F), also presenting vesicles budding from the region near the flagellar pocket (Fig. 5E)

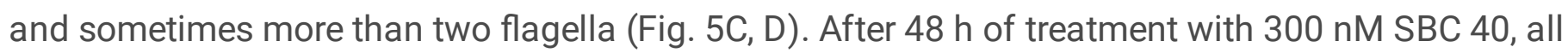
parasites were completely rounded (Fig. $5 F$ ). These results indicate the potent effect of these inhibitors to alter the morphology of promastigotes.

\subsection{SBC 39 and SBC 40 alter mitochondrion function and induce lipid bodies accumulation}

Nanomolar concentrations of SBC 39 and SBC 40 were able to reduce significantly the mitochondrial membrane potential $\left(\Delta \Psi_{m}\right)$ after $48 \mathrm{~h}$ of treatment (Fig. $\left.6 \mathrm{~A}\right)$. This effect was similar to those observed for the positive control group treated with FCCP, a mitochondrial protonophore. The readings were done 25 min after addition of $\mathrm{JC}-1$ in all groups, when both forms of the fluorochrome (monomer and $\mathrm{J}$ aggregate) are stabilized in inner portion of the mitochondrion. Although both inhibitors were able to reduce potential, only SBC 40 was able to increase significantly the ROS production at low concentrations (Fig. 6B).

Furthermore, both inhibitors were able to induce lipid bodies accumulation after $48 \mathrm{~h}$ of treatment (Fig. 7). However, for SBC 39 the concentration to increase the presence of lipid bodies was 3-times higher than those for SBC 40.

\subsection{SBC 39 and SBC 40 alters the ultrastructure of promastigotes}

Transmission electron microscopy was used to analyze the ultrastructural alterations induced by SBC 39 and SBC 40. Figure 8A shows a control promastigote presenting a structural organization without any alteration for the nucleus $(N)$, mitochondrion $(M)$, kinetoplast $(k)$, flagellum (f) and cell surface. After $48 \mathrm{~h}$ 
of treatment with $500 \mathrm{nM}$ or $1 \mu \mathrm{M} \mathrm{SBC} 39$, several alterations were observed, such as: 1) loss of the mitochondrial matrix content and vesiculation of its inner membrane (Fig. 8C, D, F); 2) presence of several large vacuoles similar to autophagosomes engulfing parts of the cytosol (Fig. 8B, E); 3), increased number of lipid bodies (Fig. 8E); and 4) disorganization of the kinetoplast (Fig. 8C-E). For the treatment with SBC 40, in concentrations much lower, the ultrastructural alterations were similar for those with SBC 39. A significant accumulation of lipid bodies randomly distributed throughout the cytosol were observed (Fig. 9C, D). The presence of glycosomes were easily observed in ultrathin sections of treatedpromatigotes, probably indicating an increase number of them, since they are difficult to observe in control parasites (Fig. 9C). Furthermore, several extracellular vesicles inside the flagellar pocket (Fig. 9D), and the presence of autophagosome-like vacuoles in close association with nucleus and mitochondrion (Fig. 9A, E, F) were observed. Alterations in the trans-Golgi network (Fig. 9E), disorganizing of the kinetoplast (Fig. 9B), and intense mitochondrial swelling (Fig. 9B, F) were also induced by the treatments.

Trying to understand better the ultrastructural effects induced by SBs, we decided to carry out treatments with high concentrations and short time of incubation. For this, we used the concentration of $5 \mu \mathrm{M}$ for just $6 \mathrm{~h}$. The results obtained indicated significant alterations showing that these new compounds present a potent activity against Leishmania. Plasma membrane projections were observed after treatment with $5 \mu \mathrm{M}$ SBC 39 (Fig. 10B-D, arrowhead). Interesting, these projections appeared frequently in regions of the membrane close to endoplasmic reticulum (Fig. 10D, arrowhead). Therefore, we decided to observe these projections by electron tomography. Figure 11A-D shows a serial section tomography of $L$. amazonensis promastigotes treated with $5 \mu \mathrm{M} \mathrm{SBC} 39$ for $6 \mathrm{~h}$. From these sections, a specific area of the parasite surface was reconstructed (Fig. 11E, F) revealing the ultrastructure of this plasma membrane projection. Images confirmed the presence of the endoplasmic reticulum profile near the projection, and the absence of microtubules associated to this projection. The alignment of the electron tomograms allows us to observe the relation between projection and endoplasmic reticulum (Suppl. Figs. 1 and 2 movies).

Furthermore, the treatment with SBC 39 and SBC 40 for short time caused several other alterations in the ultrastructure of the promastigotes such as: mitochondrial swelling with an increased numbers of mitochondrial cristae (Fig. 10B, C, F); presence of large vacuoles and myelin-like figures (Fig. 10B-F); and disorganization of kinetoplast (Figure 11E). After alignment of several electron tomograms, it is possible to observe some of these changes in a movie containing a large volume of one treated promastigote with 5 4 M SBC 39 (Suppl. Fig. 3 - movie).

\subsection{Effects of SBC $\mathbf{4 0}$ on the fine structure of intracellular amastigotes}

Transmission electron microscopy was also used to study the fine structure of intracellular amastigotes and the effects induced by the treatments. Figure 12 shows the ultrathin sections of $L$. amazonensis intracellular amastigotes treated with $1 \mu \mathrm{M} \mathrm{SBC} 40$ for $48 \mathrm{~h}$. Several alterations in the ultrastructure were observed, which are absent in the control parasites (Fig. 12A) such as: mitochondrial swelling (Fig. 12C); 
presence of autophagosome-like vacuoles containing membrane profiles (Fig. 12C-D); alterations in the plasma membrane ultrastructure (Figure 12B-D, arrowhead); and disorganization of the kinetoplast (Figure 12D). Together, these images suggest the potent effect of benzyl farnesyl amine mimetics in $L$. amazonensis intracellular amastigotes.

\subsection{Identification and quantification of the free sterol}

Analyses of the free sterol composition of control and treated Leishmania amazonensis promastigotes (Table 1) revealed that the major sterols of control (untreated) promastigotes were ergosta-5,7,24(24')-

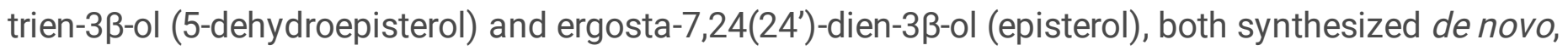
which accounted for $8 \%$ and $2 \%$, respectively, of the total sterols. While other minority sterols such as

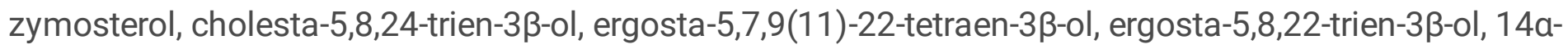

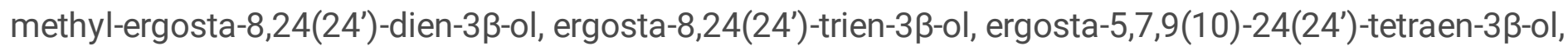

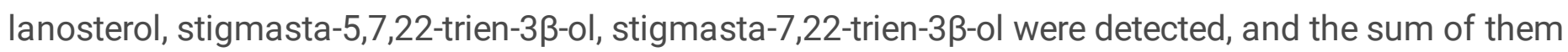
reprsented $14 \%$ (Table 1). Additionally, cholesterol, taken by endocytosis from the growth medium, accounted for $6 \%$.

Incubation of $L$. amazonensis promastigotes with increasing concentrations of SBC $39(300 \mathrm{nM}-1 \mu \mathrm{M})$ or SBC $40(30 \mathrm{nM}-300 \mathrm{nM})$ for $48 \mathrm{~h}$ induced reduction of the relative level of the main endogenous sterol 5-dehydroepisterol in about $50 \%$. However, promastigotes in presence of SBC 39 followed displayed a significant accumulation of ergosta-8, 24(24')-dien-3ß-ol (fecosterol) in $40 \%$, while in the case of SBC 40 to the highest concentration, fecosterol reached only $16 \%$ (Table 1 ). Episterol is not significantly affected. These changes in the relative percentages of endogenous sterols are compensated with an increase in the percentage of cholesterol, which account for ca. 19\% and 26\% for SBC 39 and SBC 40 respectively.

When performing the quantitative analysis of cholesterol and endogenous sterol (Table 2), it can be seen that both drugs induce a concomitant 10 and 14 - fold (from $5.12 \mu \mathrm{g} / X 10^{8}$ control cells to 0.4 or $0.5 \mu \mathrm{g} /$ $X 10^{8}$ treated cells; $\nabla 90 \%$ ) reduction of the content of endogenous sterols when compared with untreated cells. While cholesterol mass reduction is related to the decrease in cell density and not by the drugs actions.

\section{Discussion}

Although leishmaniasis has a number of treatment options, its therapy has a lot of problems as extensive toxicity, lack of efficacy, parenteral route of administration affecting compliance, high costs, and emerging drug resistance [29]. Visceral, cutaneous and mucocutaneous leishmaniasis remain some of the most devastating neglected tropical diseases. Thus, there is an urgent need for development of the new anti-leishmanial compounds that have more efficacy, low toxicity and cost, and preferentially administrated by oral or topic routes.

Sterol biosynthesis (SB) is an important metabolic pathway in Leishmania sp. For many years, several SB inhibitors have been studied against both developmental stages of the parasite in vitro [15-17, 23, 28, 


\section{Table 2}

Free and total cholesterol and endogenous sterol contents in mass $(\mu \mathrm{g})$ present in $L$. amazonensis growth in absence or presence of SBC 39 and SBC 40.

Cholesterol $(\mu \mathrm{g}) \quad$ Endogens sterol

$(\mu \mathrm{g})$

$\begin{array}{lll}\text { Control } & 0.116 & 5.12 \mu \mathrm{g} \\ 300 \mathrm{nM} & 0.078 & 2,12\end{array}$

SBC 39

$500 \mathrm{nM}$

0.062

1.18

SBC 39

$1.0 \mu \mathrm{M}$

0.052 / 2-fold less

0.54 / 10 fold less

SBC 39

$30 \mathrm{nM}$

0.108

3.56

SBC 40

$100 \mathrm{nM}$

0.078

0.92

SBC 40

$300 \mathrm{nM}$

0.054 / 2-fold less

0.38 / 14-fold less

SBC 40

30-32]. Some of them have also been tested in vivo against Trypanosoma cruzi, inducing a potent suppressive effect in models of acute Chagas' disease [30,33-35]. An important step of the SB is catalyzed by the enzyme squalene synthase (SQS). SQS is responsible for the reaction that catalyze the first committed step in the SB pathway; thus, not interfering with isoprenoid production and its metabolites [11,36]. Several classes of squalene synthase inhibitors, such as quinuclidine derivatives, have been studied as potent SB inhibitors. The first quinuclidine derivatives tested against Leishmania sp. was BPQ-OH, ER-119884 and E5700 inducing cell death in association with the depletion of the parasite's endogenous sterols [23, 31]. Furthermore, other quinuclidine derivative, WSP 1267, showed potent effect against Candida albicans, C. parapsilosis, and C. tropicalis, with a $\mathrm{MIC}_{50}$ of $2 \mu \mathrm{g} / \mathrm{ml}$ [37]. Some quinuclidine derivatives were also able to inhibit the recombinant $L$. majorSQS at submicromolar concentrations, exhibiting selectivity action for the parasite enzyme [20-21]. Benzyl farnesyl amine mimics were also reported as to be selective inhibitors of human SQS. They were explored for their potential use for development of cholesterol lowering treatment options for hypercholesterolemia in man [38]. 
In this study, we report the activity of several benzyl farnesyl amine mimetics against Leishmania amazonensis. The compounds SBC 39 and SBC 40 showed the most pronounced effects on the growth of $L$. amazonensis intracellular amastigotes, associated with a low cytotoxicity in mammal cells and a higher selectivity index of 117.7 and 180, respectively. These selectivity indexes are higher than those found after treatment with posaconazole and itraconazole, two potent inhibitors of the growth of $L$. amazonensis [16]. Herein, the antiproliferative activities of SBC 39 and SBC 40 were in the nanomolar range against both extracellular promastigotes and intracellular amastigotes. The biological activity against Leishmania amazonensis was lower than those found for E5700 and ER-119884, two SQS inhibitors from Eisai Pharmaceutical Company, previously studied against Leishmania [17, 26]. Nevertheless, it is noteworthy to mention, that further development of E5700 and ER 119884 has been stopped, because it caused testicle atrophy in a small animal experiment. Beyond this, quinuclidine derivatives often bear a certain risk of neurological side effects (neurotoxicity of antimalarial drug quinine and other quinuclidine containing drugs). Therefore, medicinal chemistry has today an increasing interest, to avoid the quinuclidine moiety in early phase drug development. Last but not least, benzyl farnesyl amine mimetics have a much lower production cost, what is especially highly attractive for antiinfectious drug design in tropical emerging countries, where cost restrictions play sometimes a limiting factor in pharmaceutical development. Thus, these results should regarded as a highly valuable contribution to SB inhibitors research in tropical parasites with a high potential for further drug development.

To analyze morphological and ultrastructural alterations, we used scanning and transmission electron microscopy, respectively. Some morphological alterations such as the swollen of the cell body and the presence of several flagella were observed. Although the number of flagella in treated promastigotes was altered, we did not observe arrest of the cell cycle or presence of multiple numbers of nucleus and kinetoplast, similar to those observed by our group with other SB inhibitors $[16,17,28]$. Transmission electron microscopy images indicated that mitochondrion ultrastructure was dramatically altered after treatment with SBC 39 and SBC 40 (Figures 8 and 9). Using fluorescence markers for mitochondrial membrane potential and ROS production, as $\mathrm{JC}-1$ and $\mathrm{H}_{2}$ DCFA respectively, it was possible to confirm the mitochondrial damage provoked by the treatments (Fig. 6). Alterations in the mitochondrion structure and function could be related to significant changes in the lipidic composition of the mitochondrial membranes, since previous studies showed that the unique and ramified mitochondrion of the trypanosomatid have a special composition of 24-methyl sterols [14]. Interestingly, in some images, several glycosomes were observed after treatment with SBC 40 (Fig. 9C), which could indicate an effort of the treated parasite to supply the mitochondrial damages and a possible decrease in the oxidative phosphorylation. An increase number of glycosomes could help the parasite to compensate the ATP production using the glycolytic pathway.

Another important alteration was observed in the plasma membrane (Fig. 5), which could be consequence of 24-methyl sterols depletion that was replaced by toxic intermediates of the sterol biosynthesis. This phenotype was also observed after treatment with several other SB inhibitors in 
Leishmania [15-17, 26-27, 38]. Moreover, several projections on plasma membrane were observed after only $6 \mathrm{~h}$ of treatment, which were better observed by electron tomography (Figure 10,11). Projections could also be related with the secretory pathway, since in some images, as shown in figure 9D, we observed several extracellular vesicles secreted by treated promastigotes. The presence of several giant vacuoles containing portions of the cytosol, damaged organelles and membranes could be related with an intense autophagic process, which could increase the secretory activity by the treated promastigotes.

After analyses of sterol composition, our results suggest that the compounds SBC 39 and SBC 40 have at least two mechanisms of action, both affecting the integrity of the plasma membrane. The first one, both act as inhibitors of the enzyme sterol $\Delta^{8} \rightarrow \Delta^{7}$ isomerase, being the SBC 39 better inhibitor. The second mechanism of action would be the blockade of endogenous sterol biosynthesis at the level of SQS, where the SBC 40 has the greatest effect.

In conclusion, our results support the notion that SBC 39 and SBC 40 are promising new chemotherapeutic agents against Leishmania sp, since they presented a very high specificity for the parasite. Furthermore, our findings justify future studies to better understand the mode of action and also using in combination therapy with other SB inhibitors as a new therapeutic strategy that could reduce toxicity, but increase efficacy of treatment.

\section{Declarations}

\section{Acknowledgement.}

Thanks to Dr. Carolina de Lima Alcântara for all contributions with electron tomography analysis. This work was supported by Fundação Carlos Chagas Filho de Amparo à Pesquisa do Estado do Rio de Janeiro [FAPERJ], Conselho Nacional de Desenvolvimento Científico e Tecnológico (CNPq) and Coordenação de Aperfeiçoamento de Pessoal de Nível Superior (CAPES).

\section{Ethical declarations.}

The experimental involving animals follows the recommendations described in the ARRIVE guidelines.

The authors declare no competing interests.

\section{References}

1. Alvar, J. et al. Leishmaniasis worldwide and global estimates of its incidence. PloS One 7, e35671 (2012).

2. Oryan, A. \& Akbari, M. Worldwide risk factors in leishmaniasis. Asian Pac. J. Trop. Med. 9, 925-932 (2016). 
3. Silveira, F.T., Lainson, R., de Castro Gomes, C.M., Laurenti, M.D. \& Corbett, C.E. 2009. Immunopathogenic competences of Leishmania (V.) braziliensis and L. (L.) amazonensis in American cutaneous leishmaniasis. Parasite Immunol. 31, 423-431 (2009).

4. Silveira, F.T., Lainson, R. \& Corbett, C.E. Further observations on clinical, histopathological, and immunological features of borderline disseminated cutaneous leishmaniasis caused by Leishmania (Leishmania) amazonensis. 2005. Mem. Inst. Oswaldo Cruz 100, 525-534 (2005).

5. Davidson, R.N. Practical guide for the treatment of leishmaniasis. Drugs 56, 1009-1018 (1998).

6. Uliana, S.R., Trinconi, C.T. \& Coelho, A.C. Chemotherapy of leishmaniasis: present challenges. Parasitol. 20, 1-17 (2017).

7. Croft, S.L., Neal, R.A., Pendergast, W. \& Chan, J.H. The activity of alkyl phosphorylcholines and related derivatives against Leishmania donovani. Biochem. Pharmacol. 36, 2633-2636 (1987).

8. Sundar, S. et al. Oral miltefosine for Indian visceral leishmaniasis. N. Eng. J. Med. 347, 1739-1746 (2002).

9. Croft, S.L., Sundar, S. \& Fairlamb, A.H. Drug resistance in leishmaniasis. Clin. Microbiol. Rev. 19, 111126 (2006).

10. van Griensven, J. et al. Combination therapy for visceral leishmaniasis. Lancet Infect. Dis. 10, 184194 (2010).

11. de Macedo-Silva, S.T., de Souza, W. \& Rodrigues, J.C.F. Sterol biosynthesis pathway as an alternative for the antiprotozoan parasite chemotherapy. Curr. Med. Chem. 22, 2186-2198 (2015).

12. Shang, N. et al. Squalene synthase as a target for Chagas disease therapeutics. PLoS Pathog. 10, e1004114 (2014).

13. Hargrove, T.Y. et al. Complexes of Trypanosoma cruzi sterol 14a-demethylase (CYP51) with two pyridine-based drug candidates for Chagas disease: structural basis for pathogen selectivity. J. Biol. Chem. 288, 31602-31615 (2013).

14. Rodrigues, J.C. \& de Souza, W. Ultrastructural alterations in organelles of parasitic protozoa induced by different classes of metabolic inhibitors. Curr. Pharm. Des. 14, 925-938 (2008).

15. de Macedo-Silva, S.T., de Oliveira Silva, T.L., Urbina, J.A., de Souza, W., Rodrigues, J.C.F. Antiproliferative, ultrastructural, and physiological effects of amiodarone on promastigote and amastigote forms of Leishmania amazonensis. Mol. Biol. Int. 2011, 8760201 (2011).

16. de Macedo-Silva, S.T., Urbina, J.A., de Souza, W. \& Rodrigues, J.CF. In vitro activity of the antifungal azoles itraconazole and posaconazole against Leishmania. PLoS One 8, e83247 (2013).

17. de Macedo-Silva, S.T, Visbal, G., Urbina, J.A., de Souza, W. \& Rodrigues, J.C.F. Potent in vitro antiproliferative synergism of combinations of ergosterol biosynthesis inhibitors against Leishmania amazonensis. Antimicrob. Agents Chemoth. 59, 6402-6418 (2015).

18. Abe, I., Tomesch, J.C., Wattanasin, S. \& Prestwich, G.D. Inhibitors of squalene biosynthesis and metabolism. Nat. Prod. Rep. 11, 279-302 (1994). 
19. Kourounakis, A.P., Katselou, M.G., Matralis, A.N., Ladopoulou, E.M. \& Bavavea, E. Squalene synthase inhibitors: an update on the search for new antihyperlipidemic and antiatherosclerotic agents. Curr. Med. Chem 18, 4418-4439 (2011).

20. Cammerer, S.B. et al. Quinuclidine derivatives as potential antiparasitics. Antimicrob. Agents Chemoth. 51, 4049-4061 (2007).

21. Orenes Lorente, S. et al. Biphenylquinuclidines as inhibitors of squalene synthase and growth of parasitic protozoa. Bioorg. Med. Chem. 13, 3519-3529 (2005).

22. Martins-Duarte, E.S., Urbina, J.A., de Souza, W. \& Vommaro, R.C. Antiproliferative activities of two novel quinuclidine inhibitors against Toxoplasma gondii tachyzoites in vitro. J. Antimicrob. Chemoth. 58, 59-65 (2006).

23. Rodrigues, J.C.F. et al. In vitro activities of ER-119884 and E5700, two potent squalene synthase inhibitors, against Leishmania amazonensis. antiproliferative, biochemical, and ultrastructural effects. Antimicrob. Agents Chemoth. 52, 4098-4114 (2008).

24. Warren, L. G. Metabolism of Schizotrypanum cruzi Chagas. I. Effect of culture age and substrate concentration on respiratory rate. J. Parasitol. 46, 529-39 (1960).

25. Souza, G. F., Cämmerer, S. B., Franco, C. H., Moraes, C. B. \& Freitas-Junior, L. H. “ N-[4[Benzyloxy]benzyl]-benzenemethaneamines with high biological activity against intracellular Trypanosoma cruzi and Leishmania infantum amastigotes". Int. J. Chem. Pharm. Sci. 4, 575-583 (2016).

26. Godinho, J.L., Georgikopoulou, K., Calogeropoulou, T, de Souza, W. \& Rodrigues, J.C.F. A novel alkyl phosphocholine-dinitroaniline hybrid molecule exhibits biological activity in vitro against Leishmania amazonensis. Exp. Parasitol. 135, 153-165 (2013).

27. Kremer, J. R., Mastronarde, D. N. \& Mclntosh, J.R. Computer visualization of three-dimensional image data using IMOD. J. Struct. Biol. 116, 71-76 (1996).

28. de Macedo Silva, S.T. et al. In vitro antileishmanial activity of ravuconazole, a triazole antifungal drug, as a potential treatment for leishmaniasis. J. Antimicrob. Chemother. 73, 2360-2373 (2018).

29. Zulfiqar, B., Shelper, T. B. \& Avery, V. M. Leishmaniasis drug discovery: recent progress and challenges in assay development. Drug Discov. Today 22, 1516-1531 (2017).

30. Urbina, J. A., Concepcion, J. L., Rangel, S., Visbal, G. \& Lira, R. Squalene synthase as a chemotherapeutic target in a Trypanosoma cruzi and Leishmania. Mol. Biochem. Parasitol. 125, 3545 (2002).

31. Rodrigues, J. C. F., Urbina, J.A. \& de Souza, W. Antiproliferative and ultrastructural effects of BPQ-OH, a specific inhibitor of squalene synthase, on Leishmania amazonensis. Exp. Parasitol. 111, 230-238 (2005).

32. Rodrigues, J. C. F., Attias, M., Rodriguez, C., Urbina, J.A. \& de Souza W. Ultrastructural and biochemical alterations induced by 22,26-azasterol, a $\Delta 24(25)$-sterol methyltransferase inhibitor, on promastigote and amastigote forms of Leishmania amazonensis. Antimicrob. Agents Chemother. 46, 487-499 (2002). 
33. Diniz, L. F., Caldas, I. S., Guedes, P. M., Crepalde, G., de Lana, M., Carneiro, C. M., Talvani, A., Urbina, J. A., Bahia, M. T. Effects of ravuconazole treatment on parasite load and immune response in dogs experimentally infected with Trypanosoma cruzi. Antimicrob. Agents Chemother. 54, 2979-2986 (2010).

34. Urbina, J. A., Payares, G., Contreras, L. M., Liendo, A., Sanoja, C., Molina, J., Piras, M., Piras, R., Perez, N., Wincker, P., Loebenberg, D. Antiproliferative effects and mechanism of action of SCH 56592 against Trypanosoma (Schizotrypanum) cruzi: in vitro and in vivo studies. Antimicrob. Agents Chemother. 42, 1771-1777 (1998).

35. Papadopoulou, M. V., Bloomer, W. D., Rosenzweig, H. S, O’Shea, I. P., Wilkinson, S. R., Kaiser, M., Chatelain, E., loset, J. R. Discovery of potent nitrotriazole-based antitrypanosomal agents: in vitro and in vivo evaluation. Bioorg. Med. Chem. 23, 6467-6476 (2015).

36. Brown, G. R., Clarke, D. S., Foubister, A. J., Freeman, S., Harrison, P. J., Johnson, M. C., Mallion, K. B., McCormick, J., McTaggart, F., Reid, A. C., Smith, G. J., Taylor, M. J. Synthesis and activity of a novel series of 3-biarylquinuclidine squalene synthase inhibitors. J. Med. Chem. 39, 2971-2979 (1996).

37. Ishida, K., Rodrigues, J. C. F., Cammerer, S., Urbina, J. A., Gilbert, I., de Souza, W., Rozental, S. Synthetic arylquinuclidine derivatives exhibit antifungal activity against Candida albicans, Candida tropicalis and Candida parapsilopsis. Ann. Clin. Microbiol. Antimicrob. 10, 3 (2011).

38. Brinkmann, J. A., Damon, R. E., Fell, J. B., Perez, L. B., Scallen, T. J., Vedananda, T. R. Squalene synthase inhibitors: isosteric replacements of the farnesyl side chain of benzyl farnesyl amine. Bioorg. Med. Chem. Let. 6, 2491-2494 (1996).

\section{Table}

Due to technical limitations, table 1 is only available as a download in the Supplemental Files section.

\section{Scheme}

Scheme 1 is not available with this version

\section{Figures}

\section{Figure 1}

Evaluation of cytotoxicity effects of SBCs on Leishmania amazonensis promastigotes. Cell viability and cytotoxicity were evaluated against promastigotes using the MTS/PMS assay after $72 \mathrm{~h}$ of treatment. The cytotoxicity concentration to reduce $50 \%$ of viable promastigotes $\left(\mathrm{CC}_{50}\right)$ was determined. SBC37, SBC39, SBC40, SBC41, SBC42, and SBC43 presented $\mathrm{CC}_{50}$ lower than $5 \mu \mathrm{M}$. Bars represent standard deviation; $* p<0.05, * \star p<0.01$, and ${ }^{* * *} p<0.001$. 
Figure 2

Evaluation of antiproliferative effects of SBCs on Leishmania amazonensis promastigotes. Parasites were treated with SBCs for $72 \mathrm{~h}$ to evaluate the parasite growth. The inhibitors were added at different concentrations after $24 \mathrm{~h}$ of growth. The most potent inhibitors were SBC37 (A), SBC39 (B), SBC40 (C), and SBC43 $(\mathrm{F})$ with $\mathrm{IC}_{50}$ of $557 \mathrm{nM}, 560.05 \mathrm{nM}, 303.78 \mathrm{nM}$, and $536.85 \mathrm{nM}$, respectively, after $72 \mathrm{~h}$ of treatment.

\section{Figure 3}

Evaluation of cytotoxicity effects of SBCs on murine macrophages. The MTS/PMS assay was used to evaluate the cytotoxicity against murine macrophages after $72 \mathrm{~h}$ of treatment. The compounds SBC37 (A), SBC38 (B), SBC39 (C), and SBC40 (D) presented low cytotoxicity to mammalian cells, with $\mathrm{CC}_{50}$ of $33.94 \mu \mathrm{M}, 40.53 \mu \mathrm{M}, 40.65 \mu \mathrm{M}$, and $39.15 \mu \mathrm{M}$, respectively. Bars represent standard deviation; ${ }^{\star} p<0.05$, $\star \star p<0.01$, and $* * \star p<0.001$.

\section{Figure 4}

\section{Evaluation of antiproliferative effects of SBC37, SBC39, and SBC40 on Leishmania amazonensis}

intracellular amastigotes. Parasites were treated with different concentrations of the inhibitors for $72 \mathrm{~h}$ to evaluate the parasite growth. After $72 \mathrm{~h}$ of treatment, the $\mathrm{IC}_{50}$ was determined for each inhibitors tested and the values were $740.48 \mathrm{nM}, 345.35 \mathrm{nM}$, and $217.5 \mathrm{nM}$, respectively. Thus, the selectivity index obtained was $45.83,117.7$, and 180 , respectively. Bars represent standard deviation; ${ }^{\star} p<0.05$, ${ }^{\star \star} p<0.01$, and $* \star * p<0.001$.

Figure 5 
Scanning electron microscopy (SEM) of L. amazonensis promastigotes treated with SBCs for $48 \mathrm{~h}$. (A) Control. (B, C) 500 nM SBC39. (D) $1 \mu \mathrm{M}$ SBC39. (E) 100 nM SBC40. (F) 300 nM SBC40. The images show several changes at morphology of the parasite. Promastigotes appeared rounded and swollen (B, C, F), with vesicles next to flagellar pocket (E). Sometimes more than two flagella could be observed (C, D).

\section{Figure 6}

Evaluation of the mitochondrial physiology and function of $L$. amazonensis promastigotes, control and treated with SBC39 and SBC40 for $48 \mathrm{~h}$. (A) Measurement of $\Delta \psi_{\mathrm{m}}$ using JC-1 marker. (B) Determination of intracellular ROS by incubating the cells with $\mathrm{H}_{2}$ DCFDA. The decrease in the $\Delta \psi_{\mathrm{m}}$ value indicates a collapse in the mitochondrial transmembrane potential when parasites were treated with SBC39 and SBC40. Treatment with $100 \mathrm{nM}$ and $300 \mathrm{nM}$ SBC40 induced a significant increase of ROS. FCCP and $\mathrm{H}_{2} \mathrm{O}_{2}$ were used as positive control. The experiments were performed three times, each time in triplicate, and the figures shown are representative of these three experiments. Bars represent standard deviation. ${ }^{\star} p<0.05, * * p<0.01$, and ${ }^{* \star *} p<0.001$.

\section{Figure 7}

Analysis of Nile Red accumulation. Fluorometric analyses indicate that there is a significant increase in Nile Red accumulation after treatment with 300 nM SBC39 and 100 nM SBC40. Bars represent standard deviation. ${ }^{\star} p<0.05,{ }^{*} p<0.01$, and ${ }^{* \star *} p<0.001$.

\section{Figure 8}

Ultrathin sections of Leishmania amazonensis promastigotes, control $(A)$ and treated with $500 \mathrm{nM}(\mathrm{B}, \mathrm{C})$ or $1 \mu \mathrm{M}$ SBC39 (D-F) for $\mathbf{4 8} \mathrm{h}$. Several alterations were observed such as: loss of the matrix content and vesiculation of the inner mitochondrial membrane (C, D, F); presence of several larges vacuoles similar to autophagosomes engulfing portions of the cytoplasm (B, E); presence of lipid bodies (E); and disorganization of the kinetoplast structure (C-E). N: nucleus; m: mitochondrion; f: flagellum; $k$ : kinetoplast; asterisks: lipid bodies; v: autofagosome-like vacuole. 
Figure 9

Ultrathin sections of Leishmania amazonensis promatigotes treated with $100 \mathrm{nM} \mathrm{SBC40}$ (A-C) or $300 \mathrm{nM}$ SBC40 (D-F) for 48h. SBC40 also induced alterations in the parasite ultrastructure such as: mitochondrial swelling and structural disorganization (A, B, F); presence of many lipid bodies eletrondense and eletronlucent (stars, C-D); high number of extracellular vesicles inside flagellar pocket (D); presence of several vacuoles similar to autophagosomes containing portions of the cytoplasm and membranes (A, E, F). Panel E shows a dilation at the trans-Golgi network. N: nucleus; $m$ : mitochondrion; $f$ : flagellum; $k$ : kinetoplast; asterisks: lipid bodies; v: autofagosome-like vacuole; g: glycosome.

Figure 10

Ultrathin sections of Leishmania amazonensis promastigotes, control (A) and treated with $5 \mu \mathrm{M}$ SBC39 (B-D) or SBC40 (E, F) for $6 \mathrm{~h}$. After a short time of incubation with the compounds, promastigotes also presented several alterations such as: mitochondrion swelling with a significant increase number of cristae (B-C, E-F); plasma membrane projections (B-D, arrowhead); disorganization of kinetoplast (E); and presence of large vacuoles and a myelin like-figure in close association with endoplasmic reticulum (B-C, $\mathrm{E}-\mathrm{F}$, arrow). N: nucleus; m: mitochondrion; f: flagellum; k: kinetoplast; asterisks: lipid bodies; v: vacuole.

\section{Figure 11}

Electron microscopy tomography and 3D reconstruction showing a projection of the plasma membrane of $L$. amazonensis promastigotes treated with $5 \mu \mathrm{M} \mathrm{SBC} 39$ for $6 \mathrm{~h}$. The images confirmed the presence of the endoplasmic reticulum profile near the projection, and the absence of microtubules associated to it.

Figure 12 
Ultrathin section of $L$. amazonensis intracellular amastigotes treated with $1 \mu \mathrm{M}$ of SBC40 for $48 \mathrm{~h}$.

Parasites treated with SBC40 appeared with drastic alterations in plasma membrane (B-D, arrowhead), mitochondrial swelling (C), presence of autofagossome-like vacuoles (C-D), and disorganization of the kinetoplast (D). N: nucleus; m: mitochondrion; f: flagellum; k: kinetoplast; v: autofagossome-like vacuole.

\section{Supplementary Files}

This is a list of supplementary files associated with this preprint. Click to download.

- Movie1.mov

- Movie2a.mov

- Movie3.mov

- Table1.docx 